\title{
Very Light Axion
}

Jihn E. Kim ${ }^{\mathrm{a}} \mathrm{b} *$

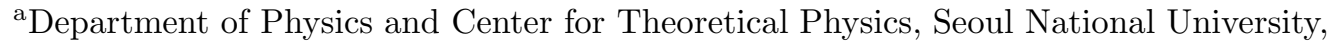
Seoul 151-742, Korea (e-mail address: jekim@phyp.snu.ac.kr)

${ }^{\mathrm{b}}$ School of Physics, Korea Institute for Advanced Study, 207-43 Cheongryangri-dong, Seoul 130-012, Korea

I review the idea of axion, axion properties, and the superstring axion.

\section{INTRODUCTION}

Quantum chromodynamics (QCD) before 1975 was described by

$\mathcal{L}=-\frac{1}{2 g^{2}} \operatorname{Tr} F_{\mu \nu} F^{\mu \nu}+\bar{q}(i \not D-M) q$

But after 1975, due to the discovery of instanton solution in non-abelian gauge theories [1], it has been known that one should consider another term also [2]

$$
\frac{\bar{\theta}}{16 \pi^{2}} \operatorname{Tr} F_{\mu \nu} \tilde{F}^{\mu \nu}
$$

which is $\mathbf{E}^{a} \cdot \mathbf{B}^{a}$ and violates the $\mathrm{CP}$ invariance in strong interactions. $|\bar{\theta}|$ is phenomenologically bounded, by the upper bound of the neutron electric dipole moment $\left|d_{n}\right|<10^{-25} e \mathrm{~cm}$,

$|\bar{\theta}|<10^{-9}$

Here, the question arises, "Why is $|\bar{\theta}|$ so small?", which is the parameter problem in the standard model. It is commonly called the strong CP problem. Most small parameters in physics have led to some ideas, mostly those related to symmetries. For example, the small ratio $M_{W} / M_{P} \ll 1$ (the so-called gauge hierarchy problem) led to supersymmetric solution [3], and $m_{u}, m_{d} \ll 1 \mathrm{GeV}$ led to the chiral symmetry 㑛.

The nicest solution of the strong CP problem is the existence of axion (very light, or invisible(?)

\footnotetext{
*This work is supported by Distinguished Scholar Exchange Program of Korea Research Foundation, KOSEF and Ministry of Education BSRI 97-2468.
}

axion). The axion is related to the Pecei-Quinn symmetry [5]. The axion relevant for the standard model VEV is the PQWW axion [6]. Since it has not been observed [7], many calculable $\bar{\theta}$ models were tried in 1978 [8]. Because the ideas without axion were turned out to be ugly, one freed the axion scale from the electroweak scale, inventing a very light (or invisible) axion 9]. For a very light axion, the model-building is one of the important aspects, since the axion decay constant is allowed within the window from SN1987A constraint and closure density of the universe [10],

$10^{9-10} \mathrm{GeV} \leq F_{a} \leq 10^{12-13} \mathrm{GeV}$

In this talk, I review why axion solves the strong CP problem, with a comment on the very light axion in superstring models.

\section{THE AXION SOLUTION}

Peccei and Quinn introduced an anomalous chiral symmetry [0], $U(1)_{A}$. If this global symmetry is spontaneously broken, there results a Goldstone boson, called axion [6]. This axion solution is a dynamical solution of the strong CP problem, in which the evolving universe settles $\bar{\theta}=0$. If the evolving universe settles $\bar{\theta}$ at $0, \bar{\theta}=0$ must be the minimum of the potential. An elegant proof of this fact has been given by Vafa and Witten 11 which is briefly repeated here. After this proof, we show how axion solves the strong CP problem.

Below the $S U(2) \times U(1)$ symmetry breaking scale, we have

$\mathcal{L}=-\frac{1}{4} F^{2}+\bar{q}\left(i \not D-M_{q}\right) q+\bar{\theta} g^{2}\{F \tilde{F}\}$ 
where $\{F \tilde{F}\}$ means $\left(1 / 32 \pi^{2}\right) F_{\mu \nu}^{a} \tilde{F}^{a \mu \nu}$, and $M_{q}$ is a diagonal, $\gamma_{5}$ free, and real quark mass matrix. We now understand that $\bar{\theta}$ is a dynamical field in axion physics, but for a moment let us treat it as a parameter. After integrating out the quark fields, we obtain the following generating functional in the Euclidian space

$$
\int\left[d A_{\mu}\right] \prod_{i} \operatorname{Det}\left(\not D+m_{i}\right) e^{-\int d^{4} x\left(\frac{1}{4 g^{2}} F^{2}-i \bar{\theta}\{F \tilde{F}\}\right)}(6)
$$

The Euclidian Dirac operator satisfies that if $i \not D \psi=\lambda \psi$ then $i \not D\left(\gamma_{5} \psi\right)=-\lambda\left(\gamma_{5} \psi\right)$. Namely, if $\lambda$ is a real eigenvalue of $i \not D$ then so is $-\lambda$, which implies

$$
\begin{gathered}
\operatorname{Det}\left(\not D+m_{i}\right)=\prod_{\lambda}\left(i \lambda+m_{i}\right) \\
=m_{i}^{N_{0}} \prod_{\lambda>0}\left(m_{i}^{2}+\lambda^{2}\right)>0
\end{gathered}
$$

where $N_{0}$ is the number of zero modes. Therefore, we obtain the following inequality using the Schwarz inequality,

$$
\begin{gathered}
e^{-\int d^{4} x V[\bar{\theta}]} \equiv \mid \int\left[d A_{\mu}\right] \prod_{i} \operatorname{Det}\left(\not D+m_{i}\right) \cdot \\
\cdot e^{-\int d^{4} x \mathcal{L}(\bar{\theta}) \mid} \\
\leq\left|\int\left[d A_{\mu}\right] \prod_{i} \operatorname{Det}\left(\not D+m_{i}\right) e^{-\int d^{4} x \mathcal{L}(\bar{\theta}=0)}\right| \\
=e^{-\int d^{4} x V[0]}
\end{gathered}
$$

which implies that

$V[\bar{\theta}] \geq V[0]$

The schematic form of $V[\bar{\theta}]$ is shown in Fig. 1, where the height of $V$ is $\left(2 Z /(1+Z)^{2}\right) f_{\pi}^{2} m_{\pi}^{2}$ with $Z=m_{u} / m_{d}$. Note that it is a periodic function of $\bar{\theta}$ with period $2 \pi$ since $Z \propto$ $\int\left[d A_{\mu}\right](\cdots) e^{i \bar{\theta} \int d^{4} x\{F \tilde{F}\}}$ and $\int d^{4} x\{F \tilde{F}\}=\mathbf{Z}$.

At this stage, any $\bar{\theta}$ will be a good theory, i.e. any nonzero value of $\bar{\theta}$ is allowed.

The axion solution is to identify $\bar{\theta}$ as a dynamical field. Interpreting it as a spin zero boson field $a$,

$\bar{\theta} \equiv \frac{a}{F_{a}}$

one should introduce a scale $F_{a}$ which is called the axion decay constant. In this axion theory,

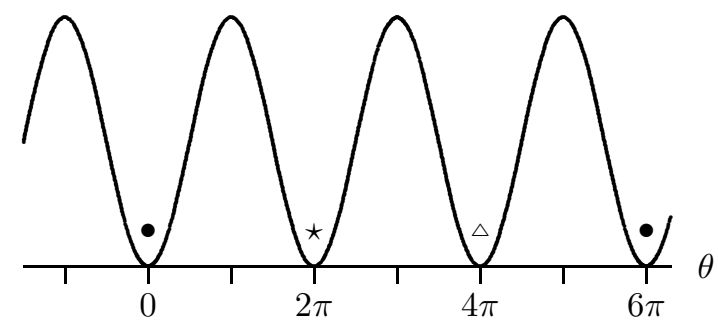

Figure 1. An axionic potential with $N_{D W}=3$. Here $\theta=a / F_{a}$.

the evolving universe allow only one stable value of $\bar{\theta}$, i.e. the minimum value of $V$ at $\bar{\theta}=0$. In this case, different $\bar{\theta}$ 's do not describe different theories, but only different vacua. An important feature of the above proof is that $\bar{\theta}$ does NOT have any potential except that coming from $\bar{\theta}\{F \tilde{F}\}$. Otherwise, the mechanism does not work.

To make $\bar{\theta}$ dynamical, one must have a mechanism to introduce a kinetic energy term for $\bar{\theta}$ and the scale $F_{a}$. Depending on this origin, one can classify axions as : (i) Goldstone boson of a spontaneously broken anomalous $U(1)_{A}\left(F_{a}\right.$ is the spontaneous symmetry breaking scale), (ii) fundamental field in string theories $\left(F_{a}\right.$ is the compactification scale), and (iii) composite field ( $F_{a}$ is the confinement scale).

The most plausible model is the superstring axion, which will be briefly commented later.

Another important parameter of the axion model is the domain wall number $N_{D W}$. It arises because in the field space all fields return to the original value after the chiral rotation (of $U(1)_{A}$ group) of $2 \pi N_{D W}$. For example, the vacua $\langle a\rangle=0,2 \pi F_{a}, 4 \pi F_{a}$, etc gives the minima of $V$, but $\langle a\rangle=0$ is identified only at $2 \pi N_{D W} F_{a} \times$ (integer).

\section{AXION PROPERTIES}

Remembering that the axion is a dynamical $\theta$ 2, we can easily derive its mass and interaction

$\overline{{ }^{2} \text { From now on we suppress }}$ - in $\bar{\theta}$ 
terms. Here, we derive the mass term only in effective field theory framework.

The simplest axion model is the heavy quark axion model since at low energy it introduces only the dynamical $\bar{\theta}$ in addtition to the standard model fields. The Lagrangian is

$\mathcal{L}=\sigma \bar{Q}_{R} Q_{L}+$ h.c. $+\cdots$

where we suppressed the Yukawa coupling constant and $\cdots$ are the other terms, including the potential respecting the $U(1)_{A}$ symmetry. The PQ global $U(1)_{A}$ symmetry is

$$
\begin{gathered}
Q_{L} \rightarrow e^{-i \frac{\alpha}{2}} Q_{L}, Q_{R} \rightarrow e^{i \frac{\alpha}{2}} Q_{R}, \sigma \rightarrow e^{i \alpha} \sigma \\
\theta \rightarrow \theta-\alpha
\end{gathered}
$$

For a nonzero VEV $\langle\sigma\rangle=F_{a} / \sqrt{2}, Q$ obtains a mass at scale $F_{a}$, the radial component $\rho$ (Higgstype field) of $\sigma$ obtains a mass at scale $F_{a}$, and at low energy there remains only the axion $a$. Thus from the kinetic term $D_{\mu} \sigma^{*} D^{\mu} \sigma$, we obtain $(1 / 2) \partial_{\mu} a \partial^{\mu} a$ where $\sigma=\left(\left[F_{a}+\rho\right] / \sqrt{2}\right) e^{i a / F_{a}}$.

Thus, below the scale $F_{a}$, the light fields are gluons and $a$ (plus the other SM fields). The relevant part of the Lagrangian respecting the symmetry (12) (i.e. with $a \rightarrow a+\alpha F_{a}$ ) is

$$
\begin{gathered}
\mathcal{L}=\frac{1}{2}\left(\partial_{\mu} a\right)^{2}+(\text { derivative term of } a) \\
+\left(\theta+\frac{a}{F_{a}}\right)\{F \tilde{F}\}
\end{gathered}
$$

Note that we created the needed $F \tilde{F}$ coupling minimally. Usually, $a$ is redefined as $a-\theta F_{a}$ so that the coefficient of $F \tilde{F}$ is $a / F_{a} \equiv \bar{\theta}$.

\subsection{Axion Mass in One Flavor QCD}

To see the essence of the mass formula, let us consider the one-flavor QCD,

$\mathcal{L}=-m_{u} \bar{u}_{R} u_{L}+$ h.c.

which possess the following hypothetical symmetry

$$
\begin{gathered}
u_{L} \rightarrow e^{i \alpha} u_{L}, \bar{u}_{R} \rightarrow e^{i \alpha} \bar{u}_{R}, m \rightarrow e^{-2 i \alpha} m \\
\theta \rightarrow \theta+2 \alpha
\end{gathered}
$$

Since $m$ is endowed with a transformation even though it is not a symmetry, Eq. (15) is useful to trace the $m$ dependence in the effective theory below the quark condensation scale $<\bar{u} u>\propto$

$$
\begin{aligned}
& v^{3} e^{i \eta / v} \\
& V=\frac{1}{2} m_{u} \Lambda^{3} e^{i \theta}-\frac{1}{2} \lambda_{1} \Lambda v^{3} e^{i(\eta / v-\theta)} \\
& -\frac{1}{2} \lambda_{2} m_{u} v^{3} e^{i \eta / v}+\lambda_{3} m_{u}^{2} \Lambda^{2} e^{2 i \theta} \\
& \quad+\lambda_{4} \frac{v^{6}}{\Lambda^{2}} e^{2 i(\eta / v-\theta)}+\cdots+\text { h.c. }
\end{aligned}
$$

where the strong interaction scale $\Lambda$ is inserted to make the dimension appropriate. Note that, for $m_{u}=0, \eta-F_{a} \theta$ can be redefined as a new $\eta$, removing the $\theta$ dependence. Thus $\theta$ is unphysical in a massless $u$-quark theory, solving the strong $\mathrm{CP}$ problem. At the minimum of the potential, the $a-\eta$ mass matrix for $m_{u} \neq 0$ is

$$
M^{2}=\left(\begin{array}{cc}
\lambda \Lambda v+\lambda^{\prime} m v, & -\lambda \Lambda v^{2} / F_{a} \\
-\lambda \Lambda v^{2} / F_{a}, & -\frac{m \Lambda^{3}}{F_{a}^{2}}+\frac{\lambda \Lambda v^{3}}{F_{a}^{2}}
\end{array}\right)
$$

Diagonalizing the above mass matrix for $F_{a} \gg$ (other mass parameters), we obtain for vacuum at $\theta=0$

$$
\begin{gathered}
m_{a}^{2}=\frac{m_{u} \Lambda}{F_{a}^{2}}\left(\frac{\lambda \lambda^{\prime} v^{4}}{\lambda \Lambda v+\lambda^{\prime} m_{u} v}-\Lambda^{2}\right), \\
m_{\eta}^{2}=\left(\lambda \Lambda+\lambda^{\prime} m\right) v
\end{gathered}
$$

which shows the essential features of the axion mass: it is suppressed by $F_{a}$, multiplied by $m_{q}$, and the rest of condensation parameters. If the above mass squared is negative, we chose a wrong vacuum and choose $\theta=\pi$ instead as the vacuum.

\subsection{Axion Mass in Two-flavor QCD}

For a realistic axion mass, we consider one family QCD, in which we assign $U(1)_{u} \times U(1)_{d}$ fictitious symmetry,

$$
\begin{gathered}
u_{L} \rightarrow e^{i \alpha} u_{L}, d_{L} \rightarrow e^{i \beta} d_{L}, m_{u} \rightarrow e^{-2 i \alpha} m_{u} \\
\quad m_{d} \rightarrow e^{-2 i \beta} m_{d}, \theta \rightarrow \theta+2(\alpha+\beta)
\end{gathered}
$$

Following the same procedure as in the previous subsection, we obtain

$$
\begin{gathered}
V=m_{u} m_{d} \Lambda^{2} \cos \frac{a}{F_{a}}-\lambda_{1} \frac{v^{6}}{\Lambda^{2}} \cos \left(\frac{2 \eta}{F_{\pi}}-\frac{a}{F_{a}}\right) \\
-\lambda_{2} m_{u} F_{\pi}^{3} \cos \left(\frac{\eta}{F_{\pi}}+\frac{\pi_{0}}{F_{\pi}}\right) \\
-\lambda_{3} m_{d} F_{\pi}^{3} \cos \left(\frac{\eta}{F_{\pi}}-\frac{\pi^{0}}{F_{\pi}}\right) \\
-\lambda_{4} m_{u} F_{\pi}^{3} \cos \left(\frac{\eta}{F_{\pi}}-\frac{\pi^{0}}{F_{\pi}}-\frac{a}{F_{a}}\right) \\
-\lambda_{5} m_{d} F_{\pi}^{3} \cos \left(\frac{\eta}{F_{\pi}}+\frac{\pi^{0}}{F_{\pi}}-\frac{a}{F_{a}}\right)+\cdots
\end{gathered}
$$


Separating two mass eigenstates, we obtain the usual axion mass formula 12

$m_{a}=\frac{m_{\pi^{0}} F_{\pi}}{F_{a}} \frac{\sqrt{Z}}{1+Z}$

where $Z=m_{u} / m_{d}$. The above formula is valid for the KSVZ model. For the PQWW and DFSZ models, one needs extra consideration, for removing the longitudinal component of $Z^{0}$. In the limit of $F_{a} \gg$ (other mass parameters), i.e. in the DFSZ model, the above formula is also valid.

\subsection{Models}

\section{KSVZ Model}

This simplest axion model was already introduced in Eqs. (11)-(13). Here we write the $U(1)_{A}$ current resulting from that Lagrangian,

$$
\begin{array}{cc}
J_{\mu} & =\tilde{v} \partial_{\mu} a-\frac{1}{2} \bar{Q} \gamma_{\mu} \gamma_{5} Q \\
& +\frac{1}{2(1+Z)}\left(\bar{u} \gamma_{\mu} \gamma_{5} u+Z \bar{d} \gamma_{\mu} \gamma_{5} d\right)
\end{array}
$$

Note that the last term results from the $a, \pi^{0}, \eta$ diagonalization procedure. There is no $a^{-}$ electron-electron coupling in this model, but can arise at loop orders.

\section{DFSZ Model}

Here one uses a singlet scalar $\sigma$, and two Higgs doublets,

$$
\begin{aligned}
& \mathcal{L}=\sigma \sigma H_{1}^{0 *} H_{2}^{0 *}-\bar{u}_{R} u_{L} H_{2}^{0 *} \\
& +\bar{d}_{R} d_{L} H_{1}^{0 *}+\bar{e}_{R} e_{L} H_{1}^{0 *}+\cdots
\end{aligned}
$$

where couplings are suppressed. This model has another parameter, i.e. the ratio of VEV's of Higgs doublets,

$x=\frac{v_{2}}{v_{1}}=\tan \beta$

This is the so-called $\left(d^{c}, e\right)$-unification model in which the electron obtains mass through the Higgs doublet giving mass to the $d$-quark. One can similarly define a $\left(u^{c}, e\right)$-unification model, and a non-unification model (a third Higgs doublet gives mass to electron). In this model, the axion mostly resides in $\sigma$, but $H_{1}^{0}$ and $H_{2}^{0}$ also contain small component of $a$. Thus, $\bar{e} i \gamma_{5} e a$ coupling arises at tree level,

$\frac{a}{F_{a}} m_{e} \frac{2 x}{x+x^{-1}} \bar{e} i \gamma_{5} e$
The axion current is given by

$$
\begin{aligned}
J_{\mu} & =\tilde{v} \partial_{\mu} a+\frac{x^{-1}}{x+x^{-1}} \sum_{i} \bar{u}_{i} \gamma_{\mu} \gamma_{5} u_{i} \\
& +\frac{x}{x+x^{-1}} \sum_{i} \bar{d}_{i} \gamma_{\mu} \gamma_{5} d_{i}+(\cdots)
\end{aligned}
$$

where $(\cdots)$ is the last term in Eq. (22), arising in the process of $<q \bar{q}>$ condensation.

Detection through cavity experiments is the relevant phenomenology for very light axions.

\section{PQWW Axion}

For the PQWW axion, $v_{1}=\sqrt{2}<H_{1}^{0}>$ and $v_{2}=\sqrt{2}<H_{2}^{0}>$ break both $U(1)_{Y}$ gauge and $U(1)_{A}$ global symmetries. Following the similar, but more complicated algebra, one obtains

$F_{a}=\frac{\sqrt{v_{1}^{2}+v_{2}^{2}}}{\left(x+x^{-1}\right) N_{g}}$

where $N_{g}$ is the family number. For $x=1$ and $N_{g}=3, m_{a} \sim 150 \mathrm{keV}$; so it does not decay to $e^{+} e^{-}$pair. For other values of $x$, the axion mass is greater. For example, for $x>30$ (which is one possible region of parameter space in MSSM model) $m_{a}>2.3 \mathrm{MeV}$. But current data prefer $x$ near 2.

Particle physics phenomenology excludes the PQWW axion. Early examples are the reactor experiments, $K^{+}$decay, $\pi^{+}$decay, etc. 13]. The argument was made more concrete later [14. Note, however, that the astrophysical bound $m_{a}<10 \mathrm{meV}\left(F_{a}>10^{9} \mathrm{GeV}\right)$ is better than these particle physics arguments. $K^{+} \rightarrow \pi^{+} a$ gives $F_{a}>10^{3-4} \mathrm{GeV}$. In $J / \psi, \Upsilon$ decays, we consider the ratio

$$
\begin{gathered}
R_{Q}=\frac{\Gamma(Q \bar{Q}) \rightarrow a \gamma}{\Gamma(Q Q) \rightarrow \mu^{+} \mu^{-}}=\frac{G_{F} m_{Q}^{2}}{\sqrt{2} \pi \alpha} . \\
\cdot\left[1-\frac{\left(\frac{\pi^{2}}{2}+8 \ln 2\right) \alpha_{s}}{3 \pi}\right] Z_{Q}^{2}
\end{gathered}
$$

where $Z_{Q}=x, x^{-1}$ for $Q=2 / 3$, and $-1 / 3$ quarks, respectively. Then B.R. $(\Upsilon \rightarrow a \gamma)=(2.0 \pm 0.7) \times$ $10^{-4} Z_{b}^{2}$, and B.R. $(\psi \rightarrow a \gamma)=(3.7 \pm 0.8) \times$ $10^{-5} Z_{c}^{2}$. On the other hand, the experimental upper bounds are B.R. $(\Upsilon \rightarrow a \gamma)<3 \times 10^{-4}$ and B.R. $(\psi \rightarrow a \gamma)<1.4 \times 10^{-5}$. Therefore, $x \sim x^{-1}$ from the above condition. The variant axion models 15] can have $Z_{b}, c \propto x, x^{-1}$, leading to a large axion mass. For $m_{a}>3 m_{e}$, the decay $\pi^{+} \rightarrow e^{+} e^{-} e^{+} \nu_{e}$ can be used to rule out shortlived "visible" axion models 14. 


\section{4. $a \gamma \gamma$ Coupling}

For the very light axion models, the axionphoton-photon coupling is represented by two pieces [16]: $\bar{c}_{a \gamma \gamma}$ which is defined by the global symmetry of the Lagrangian (i.e. term given by the short distance scale) and the piece added below the quark condensation scale (i.e. the term added in the effective theory at long distance scale),

$$
\begin{aligned}
c_{a \gamma \gamma}= & \bar{c}_{a \gamma \gamma}-\frac{2}{3} \frac{4+Z}{1+Z} \\
= & \bar{c}_{a \gamma \gamma}-1.92
\end{aligned}
$$

where we used $Z=0.6$. Since $\bar{c}_{a \gamma \gamma}$ is extracted from the coefficient of $\left(a / F_{a}\right)\left\{F_{\text {e.m. }} . \tilde{F}_{\text {e.m. }}\right\}$, one can easily convince himself that

$\bar{c}_{a \gamma \gamma}=\frac{E}{C}$

where $E=\operatorname{Tr} Q_{e m}^{2} Q_{P Q}$ and $\delta_{a b} C=\operatorname{Tr} \lambda_{a} \lambda_{b} Q_{P Q}$, and $Q_{P Q}$ is the global $U(1)_{A}$ charge given by the Lagrangian. For a fundamental representation of $S U(N)$, we define the index $\ell=1 / 2$. Thus we obtain

KSVZ :

$$
\begin{gathered}
C_{3}=-\frac{1}{2}, C_{8}=-3, \\
E_{3}=-3 e_{Q}^{2}, E_{8}=-8 e_{Q}^{2} \\
\bar{c}_{a \gamma \gamma}=\left\{\begin{array}{rc}
6 e_{Q}^{2} & \text { for } \mathbf{3}, \mathbf{3}^{*} \\
\frac{8}{3} e_{Q}^{2} & \text { for } \mathbf{8}
\end{array}\right.
\end{gathered}
$$

DFSZ :

$$
C_{3}=N_{g}, E=\frac{8}{3} N_{g} \quad \text { for }\left(d^{c}, e\right)
$$

Here $C_{x}$ and $E_{x}$ are calculated for the representation $\mathbf{x}$ and $e_{x}$ in the KSVZ is the electromagnetic charge in units of positron charge of the heavy quark $Q$ transforming as $\mathbf{x}$ of $S U(3)_{c} \cdot c_{a} \gamma \gamma$ is given in Table 1 for several interesting cases 17 .

In reality such as in superstring models, there can be many heavy quarks which carry nontrivial PQ charges. For example, superstring models have $\sim 400$ chiral fermions. Here, light fields consist of the usual 45 chiral fields, two Higgs doublets $\left(H_{1}, H_{2}\right), 12$ gauge bosons, and their superpartners. If $H_{1}$ and $H_{2}$ carry the PQ charges, then there is the aspect of DFSZ. On the other hand, the heavy fields consist of $\sigma,(3,1,1)+$ $\left(3^{*}, 1,1\right),(1,2,1)+(1,2,1)$, etc. Among these heavy fields, $\sigma$ is present. If some color triplets and antitriplets carry the PQ charges, then there exists the KSVZ aspect. Therefore, most probably both KSVZ and DFSZ effects add up and one should calculate $c_{a \gamma \gamma}$ for a specific model. Let us hope that there will appear in the future a standard superstring model where one can predict $c_{a \gamma \gamma}$ unambiguously.

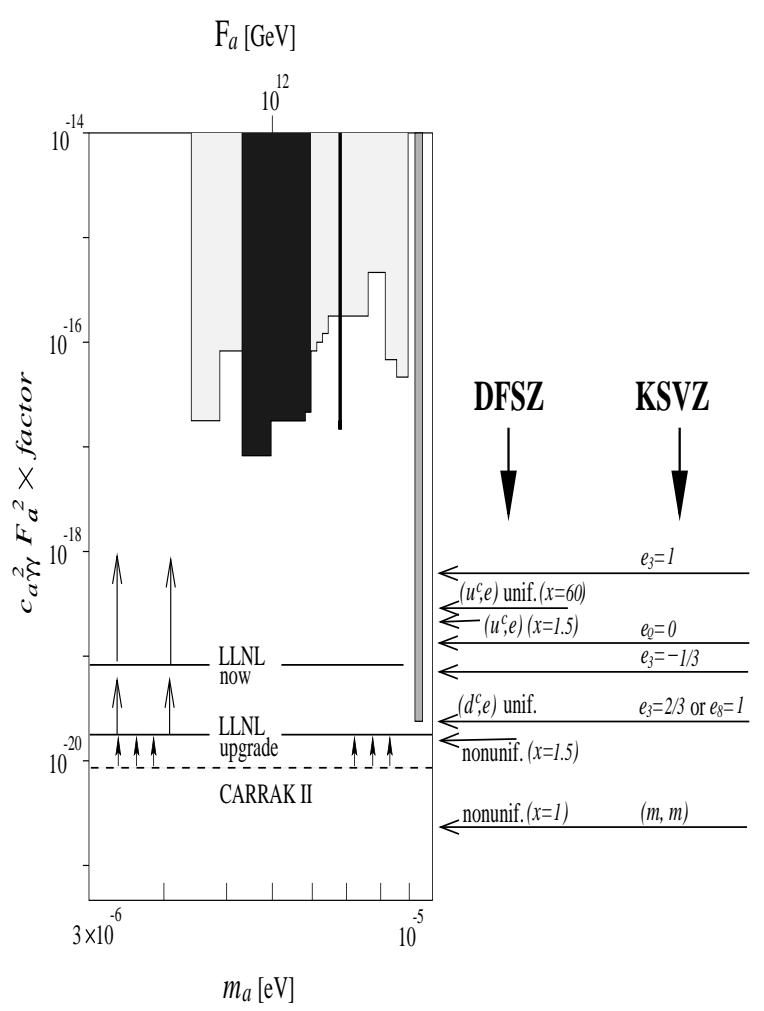

Figure 2. Data and KSVZ and DFSZ model predictions

In Fig. 2, we compare these predictions of very light axion models and cosmic axion detection experiments of RBF 18], UF [19], LLNL [20], and Kyoto [21] with several model predictions. Fig. 
Table 1

$c_{a \gamma \gamma}$ in KSVZ and DFSZ models. The unification condition in DFSZ is which Higgs doublet couples to $e$.

\begin{tabular}{rrrrr}
\hline \multicolumn{1}{l}{ KSVZ models } & & & DFSZ models \\
\cline { 1 - 2 } \cline { 5 - 5 } Heavy quark charge(s) & $c_{a \gamma \gamma}$ & & $\left(x=<H_{2}^{0}>/<H_{1}^{0}>\right)$ & $c_{a \gamma \gamma}$ \\
\hline$e_{Q}=0$ & -1.92 & & $\left(d^{c}, e\right)$ unif. $($ any $x)$ & 0.75 \\
$e_{3}=-1 / 3$ & -1.25 & & $\left(u^{c}, e\right)$ unif. $(x=1)$ & -2.17 \\
$e_{3}=2 / 3$ & 0.75 & & $\left(u^{c}, e\right)$ unif. $(x=1.5)$ & -2.56 \\
$e_{3}=1$ & 4.08 & & $\left(u^{c}, e\right)$ unif. $(x=60)$ & -3.17 \\
$e_{8}=1$ & 0.75 & & nonunif. $(x=1)$ & -0.25 \\
$\left(e_{3}=2 / 3\right)+\left(e_{3}=-1 / 3\right)$ & -0.25 & & nonunif. $(x=1.5)$ & -0.64 \\
& & nonunif. $(x=60)$ & -1.25 \\
\hline
\end{tabular}

3 is the data collection. Note that we are at the edge of finding the very light axion. One caviat in this comparison is that one uses the cosmological estimation of the axion density around earth [22].

\section{OTHER SOLUTIONS}

The strong CP problem is to understand the reason why $\theta$ is so small. There is another method to make $\theta$ small. Firstly, start $\mathcal{L}$ with vanishing $\theta$. It can be done in models with $\mathrm{CP}$ invariant Lagrangian. Second, introduce $\mathrm{CP}$ violation in weak interactions. It must be done by spontaneous $\mathrm{CP}$ violation 23]. Then, at higher orders $\theta$ will be generated, which must satisfy the experimental bound. One loop $\theta$ is typically of order $\left(10^{-4}-10^{-5}\right) \times($ couplings $)$ which is usually too large. It is therefore desirable to forbid $\theta$ even at one loopi [8]. Of course, the tree level contribution must be absent, which is implemented by making Arg.Det. $M_{q}=0$. Then calculate $\theta$ at one loop and convince oneself that the final $\theta$ is sufficiently small. Therefore, these are called calculable models.

The PQWW axion was looked for in 1978 and it seemed had troubles. Because of the trouble, the calculable models were studied extensively in 1978. But this calculable models are not as attractive as the automatic solution based on axion. Thus in 1979 the very light axion model was invented [9]. Among calculable models, the Nelson-Barr type solution 24] has been studied later, chiefly because it can mimick the Kobayashi-Maskawa CP violation at low energy. The spontaneous CP violation occurs near GUT scale, which is different from the earlier versions of calculable models using the electroweak scale as the spontaneous $\mathrm{CP}$ violation scale.

In 1985, the superstring axion was discovered, which is one of the most important theoretical reasons for the axion to exist.

\section{SUPERSTRING AXION}

If axion is the solution of the strong CP problem, it is better to be present in string models. Indeed, axion is present in any string models, but its relevance for the strong CP solution depends on the compactification scheme. Ten dimensional string models contain among massless spectrum the bosons $G_{M N}$ ( $M N$ symmetric), $B_{M N}(M N$ antisymmetric), and $\phi$, where $M, N$ run through indices $0,1, \cdots, 9$. Our interest here is the antisymmetric tensor field $B_{M N}$ which contains two kinds of axions: model-independent axion (MIa) 25] and model-dependent axions [26.

The MIa is basically $B_{\mu \nu}$ where $\mu, \nu$ is the $4 \mathrm{D}$ indices $0,1,2,3$. The dual of the field strength is defined as the derivative of MIa $a$,

$\partial^{\sigma} a \sim \epsilon^{\mu \nu \rho \sigma} H_{\mu \nu \rho}, H_{\mu \nu \rho} \sim \epsilon_{\mu \nu \rho \sigma} \partial^{\sigma} a$

The question is why we interpret this as an axion. It is due to Green, Schwarz, and Witten 27,25. The gauge invariant field strength $H$ of $B$ is $H=d B-\omega_{3 Y}^{0}+\omega_{3 L}^{0}$ with the YangMills Chern-Simmons term $\omega_{3 Y}^{0}=\operatorname{tr}\left(A F-A^{3} / 3\right)$ and the Lorentz Chern-Simmons term $\omega_{3 L}^{0}=$ $\operatorname{tr}\left(\omega R-\omega^{3} / 3\right)$. These satisfy $d \omega_{3 Y}^{0}=\operatorname{tr} F^{2}$ and

\footnotetext{
${ }^{3}$ Here, we use the differential forms.
} 
$d \omega_{3 L}^{0}=\operatorname{tr} R^{2}$. Therefore,

$d H=-\operatorname{tr} F^{2}+\operatorname{tr} R^{2}$

Note also that one had to introduce a nontrivial gauge transformation property of $B$. Then gauge anomaly is completely cancelled by introducing the so-called Green-Schwarz term [27],

$S_{G S} \propto \int\left(B \operatorname{tr} F^{4}+\cdots\right)$

which contains the coupling of the form

$$
\begin{gathered}
\epsilon_{i j K L M N O P Q R} B_{i j} F^{K L} F^{M N} F^{O P} F^{Q R} \sim \\
B_{i j}\left(\epsilon_{\mu \nu \rho \sigma} F^{\mu \nu} F^{\rho \sigma}\right)<F_{k l}><F_{p q}>\epsilon_{i j k l p q}
\end{gathered}
$$

where we note the Minkowski indices $\mu, \nu, \cdots$ and the internal space indices $i, j, \cdots$. The nonvanishing VEV $\langle F\rangle$ gives $a^{\prime} F \tilde{F}$ coupling at tree level. Thus we are tempted to interpret $a^{\prime}$ as an axion, and it was called model-dependent axion [26,28]. But we have to check that there is no dangerous potential term involving $a^{\prime}$. But it has been shown that world-sheet instanton contribution

$$
i \int_{\Sigma_{J}} d^{2} z B_{I} \omega_{i \bar{j}}^{I}\left(\partial X^{i} \bar{\partial} X^{\bar{j}}-\bar{\partial} X^{i} \partial X^{\bar{j}}\right)=2 \alpha^{\prime} B_{J}
$$

gives $a^{\prime}\left(=B_{I}\right)$ dependent superpotential [29], removing $a^{\prime}$ as a useful degree for relaxing a vacuum angle. In the above equation, $\alpha^{\prime}$ is the string tension and $\omega_{i \bar{j}}^{I}$ represents the topology of the internal space,

$B=B_{\mu \nu} d x^{\mu} d x^{\nu}+B_{I} \omega_{i \bar{j}}^{I} d z^{i} d \bar{z}^{\bar{j}}$

But $B_{\mu \nu}$ is still good since it does not get a contribution from the stringy world-sheet instanton effect. Eq. (33) implies

$\square a=-\frac{1}{M}\left(\operatorname{Tr} F_{\mu \nu} \tilde{F}^{\mu \nu}-\operatorname{Tr} R_{\mu \nu} \tilde{R}^{\mu \nu}\right)$

implying an effective Lagrangian of the form

$\mathcal{L}=\frac{1}{2}\left(\partial_{\mu} a\right)^{2}-\frac{a}{16 \pi^{2} F_{a}}\left(\operatorname{Tr} F_{\mu \nu} \tilde{F}^{\mu \nu}-\cdots\right)$

Thus $a$ is the axion (MIa). Any string models have this MIa and its decay constant is of order $10^{16} \mathrm{GeV}$ 30. At this point, we comment that there are two serious problems of the superstring axion:
(A) The axion decay constant problem-It is known that the decay constant of MIa is of order $10^{16} \mathrm{GeV}$ 30] which is far above the cosmological upper bound of $F_{a}$ [22]. A large $F_{a}$ can be reconciled with cosmological energy density if a sufficient number of radiation are added below 1 $\mathrm{GeV}$ of the universe temperature 31], but then it is hopeless to detect the cosmic axion by cavity detectors. Therefore, $F_{a}$ is better to be lowered to around $10^{12} \mathrm{GeV}$.

(B) The hidden sector problem-It is a general belief here that a hidden sector confining force, e.g. $S U(N)$, is needed for supersymmetry breaking at $10^{12} \sim 10^{13} \mathrm{GeV}$. If so, MIa gets mass also due to the $a_{M I} F^{\prime} \tilde{F}^{\prime}$ coupling where $F^{\prime}$ is the field strength of the hidden sector confining gauge field, and we expect $m_{a} \simeq \Lambda_{h}^{2} / F_{a}$ which is obviously too large to bring down MIa to low energy scale for the solution of the strong CP problem. For example, the axion gets potential both from the hidden sector scale $\Lambda_{h}$ and the QCD scale $\Lambda_{Q C D}$ (if there is no matter) in the follwing way,

$$
-\Lambda_{Q C D}^{4} \cos \left(\frac{a_{M I}}{F_{a}}+\theta^{0}\right)-\Lambda_{h}^{4} \cos \left(\frac{a_{M I}}{F_{a}}+\theta_{h}^{0}\right)
$$

where we added two terms with independent phases $\theta^{0}, \theta_{h}^{0}$ which arise at the string scale when CP is broken. Because $\Lambda_{h} \gg \Lambda_{Q C D}$, the vacuum chooses $\frac{a_{M I}}{F_{a}}+\theta_{h}^{0}=0$, i.e. $\left\langle a_{M I}>\simeq-\theta_{h}^{0} F_{a}\right.$, implying $\bar{\theta} \simeq \theta^{0}-\theta_{h}^{0}$ which is not zero in general. If we want to settle both $\theta_{h}$ and $\bar{\theta}$ at zero, then we need two independent axions. However, it is known that only MIa is available at string induced low energy physics. Therefore, we say that there is the hidden sector problem in the MIa phenomenology.

The above two problems have to be resolved if the string theory render an acceptable low energy standard model and also if the axion solution has a profound root in the fundamental theory of the universe. It turns out that it is very difficult to achieve. Only in a limited case, it may be possible to find a possible route.

One such example is the so-called anomalous $U(1)$ models [32] in which the gauge group takes the form $U(1)_{A} \times S U(3) \times S U(2) \times U(1) \times \cdots$. The gauge group $U(1)_{A}$ has an anomaly if one considers matter fields only, but in the whole theory 
there is no anomaly. The Green-Schwarz term contains

$$
\begin{aligned}
& \epsilon_{M N O P Q R S T U V} B^{M N} \cdot \operatorname{Tr} F^{O P} \\
& \cdot<F^{Q R}><F^{S T}><F^{U V}>
\end{aligned}
$$

which introduces the coupling $M_{c}\left(\partial^{\mu} a_{M I}\right) A_{\mu}$. Namely, the MIa becomes the longitudinal degree of freedom of $A_{\mu}$. Thus, the $U(1)_{A}$ gauge boson becomes massive and $a_{M I}$ is removed at low energy. Below the scale $M_{c}$, then there exists a global symmetry [33,34]. Superstring models also need an extra hidden sector confining force. Then, even if the MIa is present, it obtains a dominant contribution to the mass from the hidden sector instanton effects. One can make the contribution to the MIa mass absent if there is a massless hidden colored fermion. The first choice is the theory of a massless hidden sector gaugino without a hidden matter. But the hidden sector gaugino is NOT massless. Nevertheless, the final hidden sector gaugino mass is not introduced by hand, but it arises from the condensation of the hidden sector gauginos, thus the contribution to the MIa potential is absent in this limit. However, the string (or gravitational) theory does not preserve any global symmetry, thus there must be interactions violating the $R$ symmetry. . $^{\text {B }}$ But the contribution to the potential from the $R$ violating terms is much smaller than a naive dimensional counting. In addition, if there survives a discrete subgroup of $R$ symmetry, then the contribution to the MIa potential is sufficiently suppressed [34]. Thus, there is a hope of introducing a very light axion in superstring models.

\section{CONCLUSION}

I reviewed the very light axions from the particle theory viewpoint. The strong $\mathrm{CP}$ problem is real in QCD, 7 and as we have seen the axion solution is the most elegant one. If it exist, it can contribute to the astrophysical and cosmological evolution. If the parameters of the theory is in

\footnotetext{
${ }^{4}$ With the massless gaugino with only renormalizable gaugino self interactions, there is an $R$ symmetry.

5 There exists a comment that it is not a real problem [35], but there exists other arguments claiming that it is real.
}

the right range, it can be detected, which is the reason that this workshop is so interesting. Theoretically, on the other hand, the superstring is expected to give the low energy standard model. Therefore, if the very light axion is to solve the strong $\mathrm{CP}$ problem, it is better to be implemented in the superstring models. If the superstring derived standard model is found and it contains a very light axion, one can calculate $c_{a \gamma \gamma}$ with certainty. On the contrary if the very light axion is found, then it can lead to discovering the superstring standard model.

\section{REFERENCES}

1. A. A. Belavin, A. Polyakov, A. Schwartz, and Y. Tyupkin, Phys. Lett. B59 (1974) 85.

2. C. G. Callan, R. F. Dashen and D. J. Gross, Phys. Lett. B63 (1976) 334; R. Jackiw and C. Rebbi, Phys. Rev. Lett. 37 (1976) 172.

3. S. Weinberg, Phys. Lett. B82 (1979) 387; E. Witten, Nucl. Phys. B188 (1981) 513.

4. M. Gell-Mann, R. J. Oakes and B. Renner, Phys. Rev. 175 (1968) 2195; H. Georgi, Weak Interactions and Modern Particle Theory (Benjamin/Cummings Publishing Co., Menlo Park, CA, 1984)

5. R. D. Peccei and H. R. Quinn, Phys. Rev. Lett. 38 (1977) 1440.

6. S. Weinberg, Phys. Rev. Lett. 40 (1978) 223; F. Wilczek, ibid 40 (1978) 279.

7. R. D. Peccei, in Proc. 19th ICHEP (1978), eds. S. Homma et al. (Phys. Soc. Japan, Tokyo, 1979) p.385.

8. M. A. B. Beg and H.-S. Tsao, Phys. Rev. Lett. 41 (1978) 278; H. Georgi, Hadronic J. 1 (1978) 155; R. N. Mohapatra and G. Senjanovic, Phys. Lett. B79 (1978) 283; G. Segre and A. Weldon, Phys. Rev. Lett. 42 (1979) 1191; S. M. Barr and P. Langacker, Phys. Rev. Lett. 42 (1979) 1654.

9. J. E. Kim, Phys. Rev. Lett. 43 (1979) 103; M. A. Shifman, A. I. Vainstein and V. I. Zakharov, Nucl. Phys. B166 (1980) 493; M. Dine, W. Fischler and M. Srednicki, Phys. Lett. B104 (1981) 199; A. P. Zhitnitskii, Sov. J. Nucl. Phys. 31 (1980) 260.

10. G. Raffelt, P. Sikivie, and E. P. S. Shellard, 
in this proceedings.

11. C. Vafa and E. Witten, Phys. Rev. Lett. 53 (1983) 535.

12. W. Bardeen and S.-H. H. Tye, Phys. Lett. B74 (1978) 229.

13. T. W. Donnelly et al., Phys. Rev. D18 (1978) 1607; A. Zehnder, Phys. Lett. B104 (1981) 494; R. D. Peccei, in Proc. 19th ICHEP (Aug., 1978), eds. S. Homma et al. (Phys. Soc. of Japan, Tokyo, 1979) p. 385.

14. For a review, see for example, R. D. Peccei, in $C P$ Violation, ed. C. Jarlskog (World Scientific, Singapore, 1989) p. 503.

15. L. M. Krauss and F. Wilczek, Phys. Lett. B173 (1986) 189; W. A. Bardeen, R. D. Peccei and T. Yanagida, Nucl. Phys. B279 (1987) 401.

16. See, for example, D. B. Kaplan, Nucl. Phys. B260 (1985) 215; M. Srednicki, ibid 689.

17. J. E. Kim, Harvard preprint HUTP-98/A009 (hep-ph/9802220).

18. S. DePafilis et al., Phys. Rev. Lett. 59 (1987) 839.

19. C. Hagmann et al., Phys. Rev. D42 (1990) 1297.

20. C. Hagmann et al., Nucl. Phys. (Proc. Suppl.) B51 (1996) 1415.

21. S. Matsuki, talk presented at The 2nd Int. Workshop on Gravitation and Astrophys., ICRR of Univ. of Tokyo, 17-19 November 1997.

22. See, for example, M. S. Turner, Phys. Rev. D33 (1986) 889.

23. T. D. Lee, Phys. Rev. D8 (1973) 1226.

24. A. E. Nelson, Phys. Lett. B136 (1984) 387; S. M. Barr, Phys. Rev. Lett. 53 (1984) 329.

25. E. Witten, Phys. Lett. B149 (1984) 351.

26. E. Witten, Phys. Lett. B155 (1985) 151.

27. M. B. Green and J. H. Schwarz, Phys. Lett. B149 (1984) 117.

28. K. Choi and J. E. Kim, Phys. Lett. B165 (1985) 71.

29. X. G. Wen and E. Witten, Phys. Lett. B166 (1986) 397; M. Dine, N. Seiberg, X. G. Wen and E. Witten, Nucl. Phys. B278 (1986) 769.

30. K. Choi and J. E. Kim, Phys. Lett. B154 (1995) 393.

31. See, for example, E. D. Stewart, M. Kawasaki and T. Yanagida, Phys. Rev. D74 (1996) 6032 .

32. M. Dine, N. Seiberg and E. Witten, Nucl. Phys. B289 (1987) 589; J. Atick, L. Dixon and A. Sen, ibid, B292 (1987) 109; M. Dine, I. Ichinoise and N. Seiberg, ibid, B293 (1987) 253.

33. J. E. Kim, Phys. Lett. B207 (1988) 434; E. J. Chun, J. E. Kim and H. P. Nilles, Nucl. Phys. B370 (1992) 105.

34. H. Georgi, J. E. Kim and H. P. Nilles, Harvard preprint HUTP-98/A024 (hep-ph/9805510).

35. S. Okubo and R. E. Marshak, Phys. Rev. D87 (1992) 1059. 